\title{
Investigating the Nanoparticles Penetration Efficiency through Horizontal Tubes Using an Experimental Approach
}

\author{
Zhaoqin Yin and Zhongping Dai \\ College of Metrology and Measurement Engineering, China Jiliang University, Hangzhou, Zhejiang 310018, China \\ Correspondence should be addressed to Zhaoqin Yin; yinzq@cjlu.edu.cn
}

Received 6 October 2014; Revised 19 January 2015; Accepted 2 February 2015

Academic Editor: Alina Adriana Minea

Copyright (c) 2015 Z. Yin and Z. Dai. This is an open access article distributed under the Creative Commons Attribution License, which permits unrestricted use, distribution, and reproduction in any medium, provided the original work is properly cited.

\begin{abstract}
It is a complex transfer process of nanoparticles in a tube. In this paper, in order to quantify the penetration efficiency of nanoparticles in different flows condition through horizontal tubes, the experiments have been carried out with particles diameter between $6 \mathrm{~nm}$ and $560 \mathrm{~nm}$ in various lengths of sampling tube. The results were in good agreement with the theory of Gormley and Kennedy and the experiment results of Kumar et al. for particles size smaller than $100 \mathrm{~nm}$. Particles penetration rate increases with increasing of the Schmidt number (Sc), and it decreases with increasing Reynolds and tube length. Particles deposition on the wall induces the changes of the mass and average diameter of particles continuously. Therefore, a nondimensional parameter $(\varsigma)$ defined dependency on Reynolds number and particle residence time in tube has been used to express total mass penetration efficiency and mean size growth rate through a straight tube.
\end{abstract}

\section{Introduction}

Nanoparticles deposition inside tubes occurs in many important technological problems in biology, immunology, crystallization, and colloid and polymer science, such as enhanced heat transfer in heat exchangers [1], toxic particle transport in human lung contamination [2], pollutant particle emission from coal combustion in power plants, and the rail tube of a moving car [3]. For badly stable suspension of microsized particles, nanoparticles suspended inside a tube may coagulate or deposit on the wall due to various mechanisms that may act in combination with Brown motion and fluid turbulence [4]. The process of coagulation causes the particle clusters to grow up, and then the dynamic characteristics of large clusters are different from that of small clusters. There are five main mechanisms which may lead to particle losses on the surface of a tube, that is, gravitation, thermophoresis, electrostatics, inertial impaction, and diffusion [5]. The diffusion, inertial impaction, and turbulence are most important for deposition process. When nanoparticles deposit on the tube wall, the characteristics of the delivery particles such as number and mass concentration, average diameter, and size distribution will be changed. However, the mechanism of deposition and evolution of the nanoparticles still remains to be clarified.
In recent years the transport and deposition processes of nanoparticles in tube are of increasing concern since nanoparticles are more toxic and diffusible than larger particles. The analyses of the forces acting on nanoparticle in flow and its deposition processes have been made by many researchers [6-8]. The research of particle deposition has been studied, such as in bends [6] and a rotating curved tube [9], respectively. Numerous authors have developed theoretical expression for aerosol penetration fraction through a smooth or rough tube in laminar flow [10]. Several experimental validations of these theories have been carried out for aerosols particles with diameters smaller than $100 \mathrm{~nm}$ [11]. For turbulent tube flow, the deposition onto tube surfaces is more complicated and equations describing it cannot be solved explicitly, and these studies give very different results $[8,12]$.

The number penetration efficiency through a tube $\eta_{N}$ is defined as the ratio of outlet number concentration to inlet concentration, which is

$$
\eta_{N}=\frac{N_{\text {outlet }}}{N_{\text {inlet }}}
$$

where $N$ is the number concentration per cubic meter. 


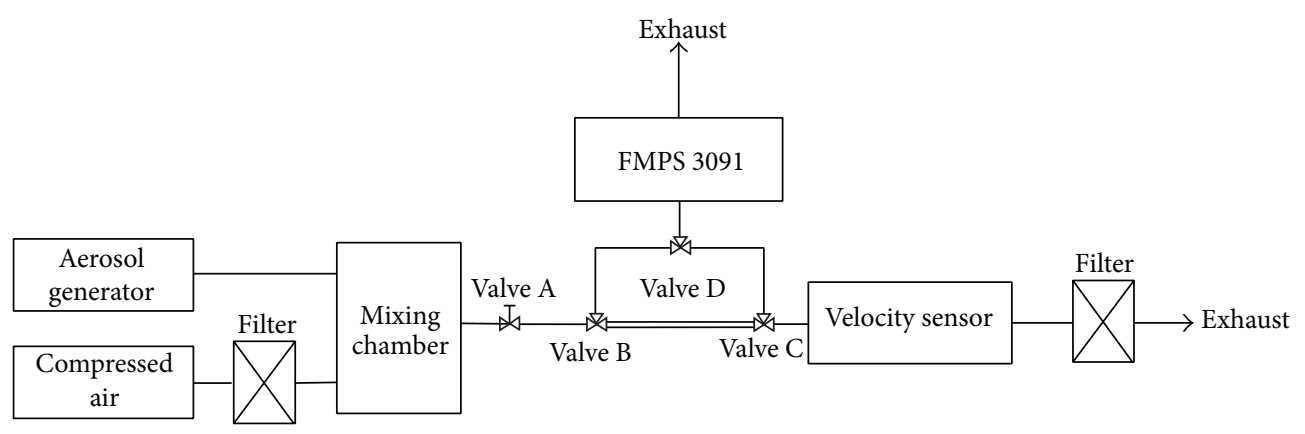

FIGURE 1: A sketch of the experimental apparatus.

Gormley and Kennedy [10] firstly developed laminar flow through a straight cylindrical tube as a function of a dimensionless deposition coefficient of particle sizes ranging from 3 to $50 \mathrm{~nm}$. The well-known equation is

$$
\begin{array}{r}
\eta_{N}=1-2.56 \xi^{2 / 3}+1.2 \xi+0.177 \xi^{4 / 3} \quad(\text { if } \xi<0.02), \\
\eta_{N}=0.819 e^{-3.657 \xi}+0.097 e^{-22.3 \xi}+0.032 e^{-57 \xi} \\
(\text { if } \xi>0.02),
\end{array}
$$

where $\xi=D \tau /(d / 2)^{2}, d$ is the tube inner diameter, and $\tau$ is the flow residence time in the tube. $D$ is the diffusion coefficient, given by Stokes-Einstein equation:

$$
D=\frac{k T C_{c}}{3 \pi \mu D p},
$$

where $k\left(=1.38 \times 10^{-23} \mathrm{~J} \mathrm{~K}^{-1}\right)$ is Boltzmann's constant, $T$ is the absolute temperature, $\mu$ is the viscosity of air, $C_{c}$ is the slip correction factor, and $D p$ is the aerodynamic diameter of particles. Allen and Raabe [13] developed the slip correction factor for solid particles for all size ranges, which is

$$
C_{c}=1+\frac{\lambda}{D p}\left[2.34+1.05 \exp \left(-0.39 \frac{D p}{\lambda}\right)\right],
$$

where $\lambda$ is the particle mean free path.

Hinds [14] simplified the equation, and Ramamurthi et al. [15] and Alonso et al. [16] confirmed the accuracy of the above equation. Noble et al. [17] measured particles in a continuous field measurement for laminar flow using scanning mobility particle sizers (SMPS) (TSI Inc.) and aerodynamic particle sizer (APS) (TSI Inc.) through a $3 \mathrm{~m}$ long aluminium tube with slight bends for connections to the sampling instruments. Their results show that the penetration efficiency was substantially smaller than the theoretical penetration efficiency.

When Reynolds number is larger than 2300, the flow is in the turbulent tube flow condition. The deposition on tube surface is more complicated than a laminar flow. Lee and Gieseke [12] presented a penetration empirical equation of particles through a tube considering diffusion, inertial impaction, and turbulence, which is

$$
\eta_{N}=\exp \left(\frac{-8 v_{d} l}{d \bar{v}}\right)
$$

where $v_{d}$ is diffusive deposition velocity through the laminar sublayer for turbulent flow in a tube, $v_{d}=$ $\left(0.04 \bar{v} / \operatorname{Re}^{1 / 4}\right)\left(\rho_{g} D / \nu\right)^{2 / 3}, l$ is the tube length, and $\rho_{g}$ is the density of the particle. Re $=\bar{v} d / \nu$ is the flow Reynolds number based on the pipe diameter and average flow velocity $\bar{v}=4 Q / \pi d^{2}$ and $Q$ is the fluid volumetric flow rate in tube.

Equation (8) is only significant for particles larger than $100 \mathrm{~nm}$. Kumar et al. [11] studied particles $(D p<100 \mathrm{~nm})$ losses in sampling tube of various lengths on typical diesel car exhaust emissions and compared penetration efficiencies with particle loss models of laminar and turbulent flows. Their results show that when the flow is laminar, the turbulent penetration model of Lee and Gieseke [12] is better than the laminar model of Hinds [14].

Since the results of the previous studies are incompatible and insufficient, the mechanisms of the nanoparticle deposition in tube should be clarified more deeply for its important application. Therefore, the aim of this study is to investigate the nanoparticles mass and number concentration penetration efficiency through different flow conditions tubes using an experimental approach.

\section{Experimental Techniques}

Ignore thermophoresis and electrostatics effect on nanoparticles and coagulation process. Assume that only diffusion, inertial impaction, and turbulence have the impact on deposition process. Experiments were made under different flow conditions. A sketch of the experimental setup is shown in Figure 1. The nanoparticles are generated by aerosol generator. The compressed air has been cleaned by a filter. Particles and compressed air have been mixed in a pressure chamber and placed twenty-four hours for mixture uniform in the vessel. The flow required for the tube is regulated using a valve downstream of the mixing chamber. In order to obtain a fully developed flow profile, there is a sufficient length between valve $A$ and valve $B$. Between valve $B$ and valve $C$ and valves $B, C$, and $D$ there are three-way valves used to regulate the fluid flow rate and direction. When the fluid passes through valves A, B, and D and a fast mobility particle sizer (FMPS3091, TSI Inc.), the entrance parameters are measured. When the aerosol passes through valves $\mathrm{A}$ and B, the sampling tube, valve C, valve D, and FMPS3091, the exit parameters are measured. The sampling tube is made of 


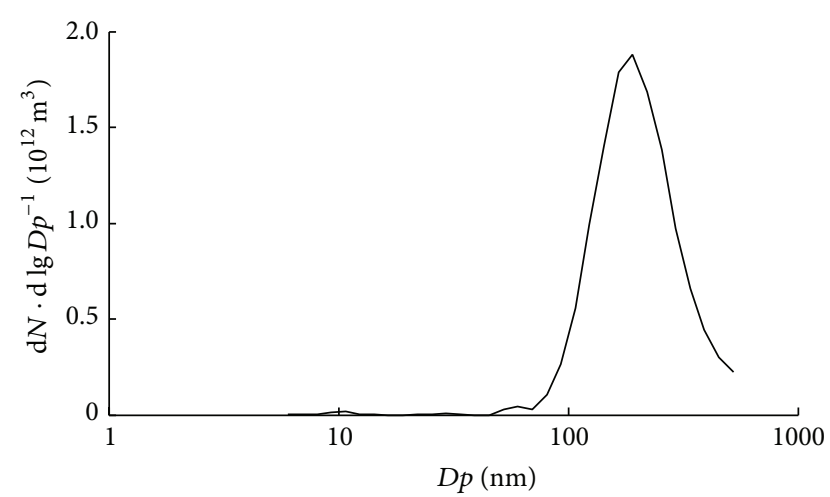

Figure 2: Particles size distribution for experiments.

plexiglass and measurements are carried out in the laboratory with temperature of $20^{\circ} \mathrm{C}$.

The FMPS3091 system uses an electrical mobility detection technique to measure particle size spectra, which has been widely applied to quantify particle size and number distribution $[18,19]$. FMPS measures particles distributions in $6-560 \mathrm{~nm}$ size range using 32 channels (16 channels per decade of size) and enables rapid determination of particle size distribution. FMPS consists of three components, (1) a bipolar radioactive charger for charging the particles, (2) a differential mobility analyzer for classifying particles by electrical mobility, and (3) multiple, low-noise electrometers for particle detection. FMPS can provide a good time resolution to measure particle number concentrations and size distributions in $1 \mathrm{~s}$. During the experiments, the sampling frequency is $1 \mathrm{~Hz}$ and is continuous for 1 minute in each measurement. The sampling flow rate is $0.01 \mathrm{~m}^{3} / \mathrm{min}$, which is $3.3 \mathrm{~m} / \mathrm{s}$ corresponding to the tube inner diameter of $8 \mathrm{~mm}$. So the experimental velocity must be larger than this value.

The LaVision Aerosol Generator generates polydisperse particles by atomizing vegetable oil into particles. The particles larger than $560 \mathrm{~nm}$ have been filtered by FMPS. To ensure accurate measurement, these experiments have been repeated at least three times with essentially the same results. Figure 2 shows the nanoparticles size distribution at air supplied pressure of 7 bars. The geometric mean size of particles is $80 \pm 5 \mathrm{~nm}$.

\section{Results and Discussion}

3.1. Parameters. Three main mechanisms including gravitational setting, molecular diffusion, and turbulent dispersion are responsible for deposition of the particle on the wall of the tube. The deposition fraction for a straight tube depends on the geometry of the test section (pipe internal radius $d$ and length $l$ ), the characteristics of air flow and aerosol particles (the volumetric flow rate $Q$, kinematic viscosity of air $v$, the particle diameter $D p$, density $\rho_{p}$, and number concentration $N)$, and the diffusion coefficient of aerosol particle in air (D). Thus the penetration efficiency is a function of different parameters. However, through dimensional analysis using Buckingham Pi theorem, we can get $p=f(\operatorname{Re}, \mathrm{Sc}, \tau)$, where

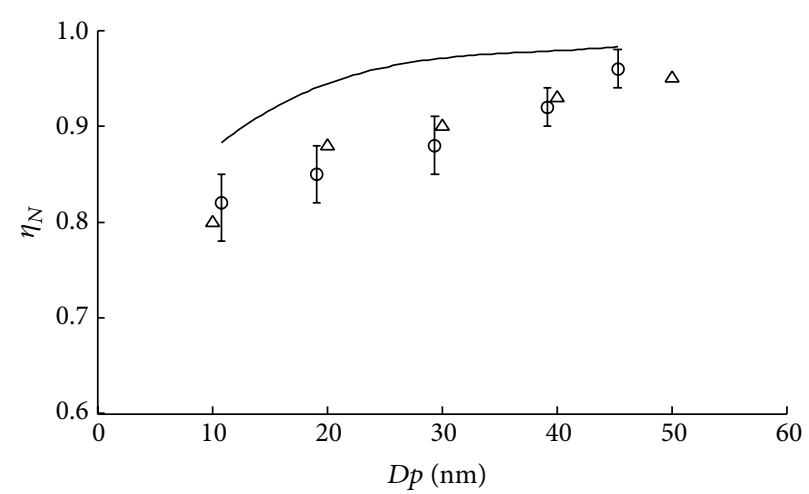

- Gormley and Kennedy $\quad$ Experimental data

FIGURE 3: Penetration efficiencies for different particle size.

Sc $=v / D$ is the Schmidt number, $\tau=l / v_{p} \cdot \bar{v} / d$ is the dimensionless particle flow residence time in the tube, and $v_{p}$ is the velocity of particle passing through the tube.

If the volume concentration of particles in the flow is very small $(<0.0001 \%)$, we can assume the motion of particles is affected by the air flow but the air flow is not affected by the particles. In the present experiments, the volumetric concentration of particles is of the order of $10^{-9}$. Thus, it is reasonable to assume that the flow is not influenced by particles and particles interactions including nucleation and coagulation are negligible.

3.2. Nanoparticles Number Penetration Efficiency through Tubes. The Brownian motion is the main mechanism leading to deposition on tube surface of particle-laden multiphase laminar flow (when $\mathrm{Re}<2300$ ). Particle losses for various cases are compared below with diffusion models for laminar, which is represented by (2a) and (2b). A comparison of the penetration efficiencies $\eta_{N}$ through tubes is shown in Figure 3 as a function of the particle diameter. In this figure, the data points with error bars are the measured penetration efficiencies through tubes for $\tau=375$ and $\operatorname{Re}=1700$. The previous similar condition experimental results are shown using triangle. The lines represent the number penetration efficiencies through the straight tube predicted by (2a) and (2b). Because of only concerning Brownian diffusion and ignoring the surface effect, the theorical results of Gormley and Kennedy show higher penetration efficiencies than the experimental results of Kumar et al. and this paper. So from Figure 3, a good agreement is obtained between this paper and the previous work. The penetration efficiency through the tube has been found to increase with increasing particle size. The losses efficiencies for particles below $20 \mathrm{~nm}$ are more than $10 \%$ stronger than the larger size particles due to the higher diffusivity of smaller particles.

Figure 4 shows the particles penetration efficiency changes with the Reynolds number at $\tau=375$. With Reynolds number increasing, the flow turns from being laminar to turbulent. The result from Figure 4 indicates with the increasing of Reynolds number the particles penetration 


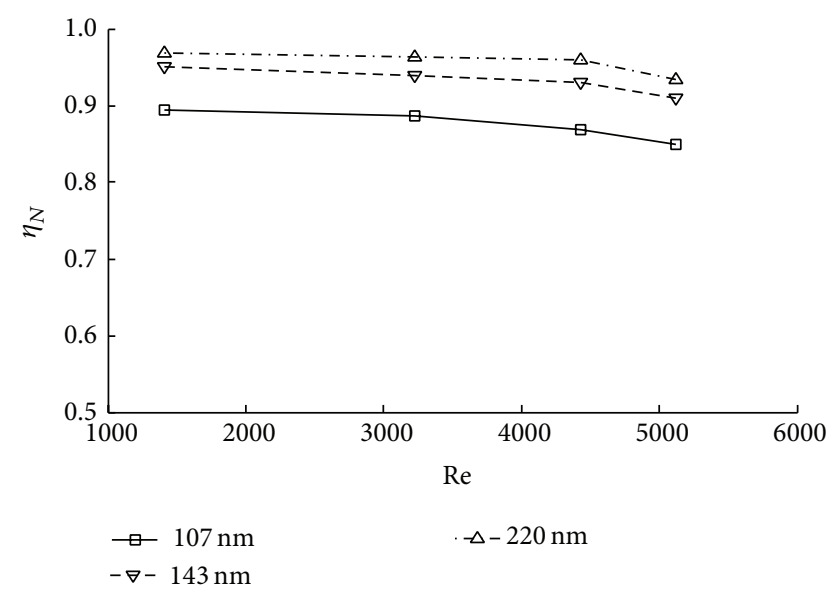

Figure 4: Penetration efficiencies for different Re at $\tau=375$.

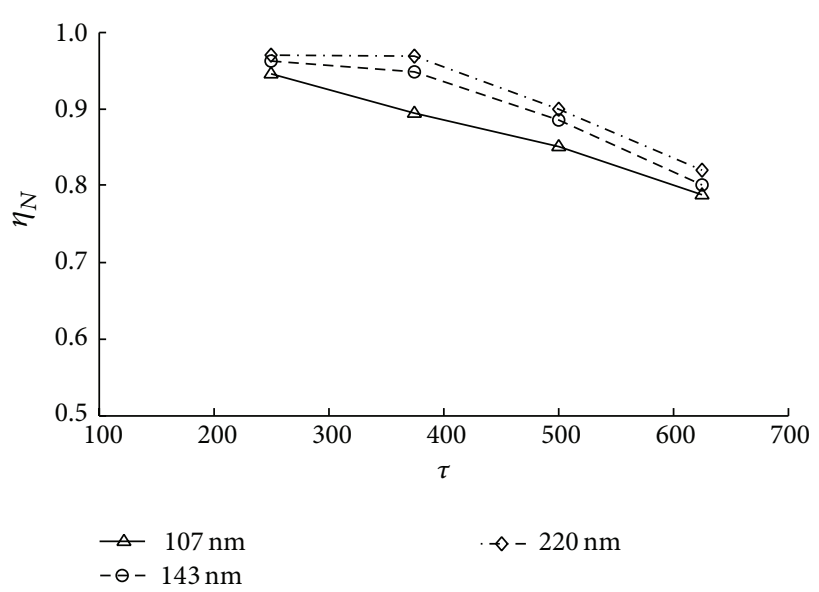

FIGURE 5: Penetration efficiencies for different $\tau$ at $\operatorname{Re}=1412$.

efficiency declines because both Brownian and turbulent motions dominate the particle diffusion in turbulence flow. The turbulence diffuser enhances particle deposition process, especially for larger particles in long tube. But most of particles larger than $100 \mathrm{~nm}$ have high penetration efficiency $(>90 \%)$ in the tube with $\tau=375$.

Figure 5 shows the particles penetration efficiency for different $\tau$ at $\operatorname{Re}=1412$. The result means the change of penetration efficiency is obvious with the tube length. Large $\tau$ means particles have more residence time in pipe and have more opportunities to deposit on the surface. As the tube length increases, the loss of particles in tube surface increases. There is near $10 \%$ increment of deposition rate when the dimensionless residence time increasing from 375 to 625 .

Figure 6 indicates the particles penetration efficiency changes with the Schmidt number. Schmidt number characterizes the ratio of mass diffusion and convection process of nanoparticles, which is in the range from 51.7 to 172573 when particle size changes from $6 \mathrm{~nm}$ to $523 \mathrm{~nm}$. The particles penetration efficiency is almost constant for the large Schmidt number but increases with Schmidt number growth when

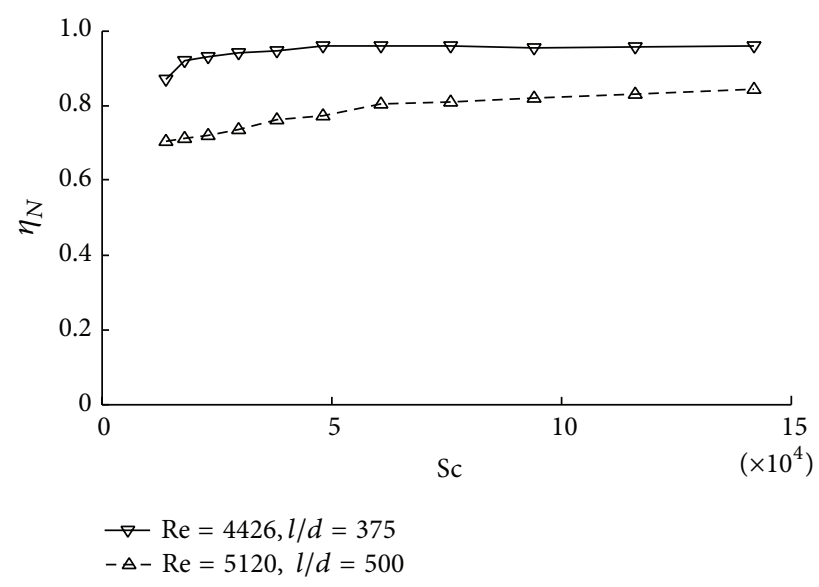

Figure 6: Penetration efficiencies for different Sc.

Sc $<5 \times 10^{4}$. Particle losses are very significant as high as $35 \%$ with particle diameter being $100 \mathrm{~nm}$.

3.3. Change of the Particle Parameters through a Tube. There are lots of functions about the particles penetration efficiency of number concentration, but few studied the mass concentration and average diameter of particles. In this part, the change of particles mass and average diameter will be discussed.

Particles deposition will reduce the mass concentration outlet of the tube. The mass penetration efficiency through a tube can be expressed as

$$
\eta_{M}=\frac{C_{\text {outlet }}}{C_{\text {inlet }}},
$$

where $C_{\text {outlet }}$ and $C_{\text {inlet }}$ are the outlet total mass concentration and inlet total mass concentration of the tube, respectively.

The total mass concentration $C$ in the experiment is expressed as follows:

$$
C=\rho_{g} \sum_{i=1}^{32} \frac{\pi D p_{i}^{3} N_{i}}{6},
$$

where $N_{i}$ is the particle number concentration per cubic meter of $i$ th channel.

Analysis of (2a), (2b), (5), and Figure 5, the length and diameter of the sampling tube, the particle diameter and the sample flow rate, and the mean velocity are variables affecting the particle losses when temperature keeps constant. So a new nondimensional parameter $\varsigma$ is used to describe the particles total mass concentration loss in straight tube which is defined as follows:

$$
\varsigma=\operatorname{Re} \cdot \tau
$$

Figure 7 shows nanoparticles total mass concentration penetration efficiency through tubes including laminar and turbulence flow with $\varsigma$ changing. A new function of $\eta_{M}$ and $\varsigma$ is

$$
\eta_{M}=-7 \times 10^{-8} \varsigma+0.974
$$




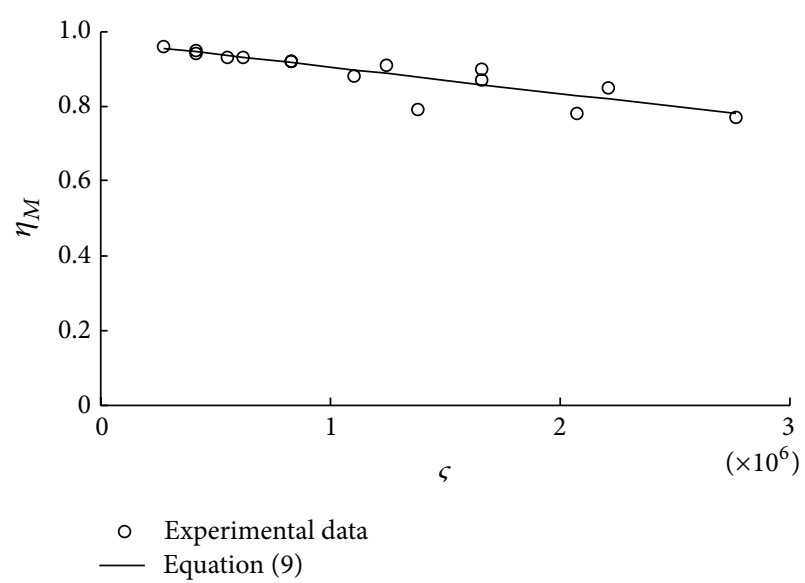

FIgURE 7: Change of the particles total mass concentration.

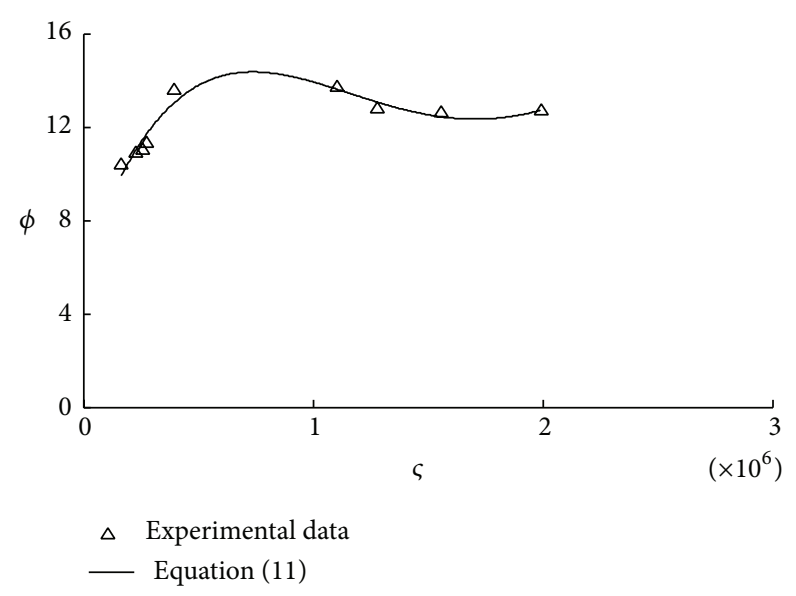

FIGURE 8: Change of the particles mean size growth rate.

Figure 7 shows the well agreement of curve and data in the experimental conditions of Reynolds number ranging from 640 to 5120 and particle residence timefrom 250 to 500 .

According to Figures 3, 4, and 5, the nanoparticles deposit on the tube wall is affected by particle size, Reynolds number, and tube length, and the small particles $(<50 \mathrm{~nm})$ are easy to deposit on the surface. As a result, the moving particles mean size will increase during the delivery process. So the particle mean size growth rate $(\varphi)$ is defined as

$$
\phi=\frac{\left(\bar{D} p_{g}\right)_{\text {outlet }}-\left(\bar{D} p_{g}\right)_{\text {inlet }}}{\left(\bar{D} p_{g}\right)_{\text {inlet }}} \times 100 \%,
$$

where $\bar{D} p_{g}$ is the particle geometric mean size.

Figure 8 shows the change of particle geometric mean size growth rate according to $\varsigma$. The particles average diameter of the tube outlet will always be larger than the inlet position. But the particle mean size growth rate $\varphi$ is fluctuation with flow condition changing. $\varphi$ increases with $\varsigma$ in laminar flow. The reason is that Brownian diffusion dominates the particles deposition process. The particles smaller than geometric mean size are easy to deposit on the tube surface. As a result the particles mean size increases and with tube length increasing $\varphi$ increases. When Reynolds number increases, the flow is in turbulence condition. Both of Brown motion and turbulence diffusion have effect on the particles. Some of the particles are larger than geometric mean size affected by turbulence diffusion deposition on the tube surface which make the particles mean size decrease. On the other hand the particles smaller than $50 \mathrm{~nm}$ are affected by Brownian diffusion deposition which make the particles mean size increase. The small particles are easy to deposit on tube surface compared to the large one, and the turbulence effect is complex, so $\varphi$ will fluctuate with $\varsigma$ in turbulence flow. The regression equation with experimental conditions is

$$
\phi=6\left(\varsigma \times 10^{-6}\right)^{3}-20\left(\varsigma \times 10^{-6}\right)^{2}+20\left(\varsigma \times 10^{-6}\right)+6.8 .
$$

\section{Conclusions}

In this study, penetration efficiency of nanoparticles (6$560 \mathrm{~nm}$ ) has been experimentally studied in different flow conditions ( $R e$ in the range of 1412-5120) in horizontal tubes of various length. Measurements were carried out in an experimental setup involving FMPS3091.

It has been found that particle losses for nanosized particle-laden multiphase tube flow are affected by particle diameter, tube length, and flow pattern. Penetration efficiency of nanoparticles through horizontal tubes can be expressed by Schmidt number, Reynolds number, and particle residence time in tube. Particles penetration rate increases with increasing of the Schmidt number, and it decreases with increasing Reynolds and tube length. The turbulent flow structures of the tube play an important role in the mechanisms and magnitude of the deposition of the nanoparticle especially for particle size larger than $100 \mathrm{~nm}$, while the losses of particles smaller than $50 \mathrm{~nm}$ are more obvious. For a better understanding of the transfer mechanisms and particles size distribution, the expressions of total mass concentration penetration efficiency and particle average size growth rate dependency on Reynolds number and particle residence time in tube have been acquired in this study.

\section{Conflict of Interests}

The authors declare that there is no conflict of interests regarding the publication of this paper.

\section{Acknowledgment}

This work is supported by National Natural Science Foundation of China (no. 11402259).

\section{References}

[1] Y. H. He, Y. S. Jin, H. Chen, Y. L. Ding, D. Q. Cang, and H. $\mathrm{Lu}$, "Heat transfer and flow behaviour of aqueous suspensions of $\mathrm{TiO}_{2}$ nanoparticles (nanofluids) flowing upward through a vertical pipe," International Journal of Heat and Mass Transfer, vol. 50, no. 11-12, pp. 2272-2281, 2007. 
[2] S. Le Guellec, D. Le Pennec, S. Gatier et al., "Validation of anatomical models to study aerosol deposition in human nasal cavities," Pharmaceutical Research, vol. 31, no. 1, pp. 228-237, 2014.

[3] A. Held, "Spectral analysis of turbulent aerosol fluxes by Fourier transform, wavelet analysis, and multiresolution decomposition," Boundary-Layer Meteorology, vol. 151, no. 1, pp. 79-94, 2014.

[4] R. Guichard, A. Tanière, E. Belut, and N. Rimbert, "Simulation of nanoparticle coagulation under Brownian motion and turbulence in a differential-algebraic framework: developments and applications," International Journal of Multiphase Flow, vol. 64, pp. 73-84, 2014.

[5] I. Mammarella, Ü. Rannik, P. Aalto, P. Keronen, T. Vesala, and M. Kulmala, "Long-term aerosol particle flux observations. Part II: particle size statistics and deposition velocities," Atmospheric Environment, vol. 45, no. 23, pp. 3794-3805, 2011.

[6] O. Ghaffarpasand, F. Drewnick, F. Hosseiniebalam et al., "Penetration efficiency of nanometer-sized aerosol particles in tubes under turbulent flow conditions," Journal of Aerosol Science, vol. 50, pp. 11-25, 2012.

[7] K. Kusdianto, M. Gen, and I. W. Lenggoro, "Area-selective deposition of charged particles derived from colloidal aerosol droplets on a surface with different hydrophilic levels," Journal of Aerosol Science, vol. 78, pp. 83-96, 2014.

[8] J. Z. Lin, Z. Q. Yin, F. J. Gan, and M. Z. Yu, "Penetration efficiency and distribution of aerosol particles in turbulent pipe flow undergoing coagulation and breakage," International Journal of Multiphase Flow, vol. 61, pp. 28-36, 2014.

[9] J. Z. Lin, P. F. Lin, and H. J. Chen, "Nanoparticle distribution in a rotating curved pipe considering coagulation and dispersion," Science China: Physics, Mechanics \& Astronomy, vol. 54, no. 8, pp. 1502-1513, 2011.

[10] P. G. Gormley and M. Kennedy, "Diffusion from a stream flowing through a cylindrical tube," Proceeding of the Royal Irish Academic, vol. 52, pp. 163-169, 1949.

[11] P. Kumar, P. Fennell, J. Symonds, and R. Britter, “Treatment of losses of ultrafine aerosol particles in long sampling tubes during ambient measurements," Atmospheric Environment, vol. 42, no. 38, pp. 8819-8825, 2008.

[12] K. W. Lee and J. A. Gieseke, "Deposition of particles in turbulent pipe flows," Journal of Aerosol Science, vol. 25, no. 4, pp. 699-709, 1994.

[13] M. D. Allen and O. G. Raabe, "Slip correction measurements of spherical solid aerosol particles in an improved Millikan apparatus," Aerosol Science and Technology, vol. 4, no. 3, pp. 269286, 1985.

[14] W. C. Hinds, Aerosol Technology: Properties Behavior and Measurement of Airborne Particles, John Wiley \& Sons, Chichester, UK, 1999.

[15] M. Ramamurthi, R. Strydom, and P. K. Hopke, "Assessment of wire and tube penetration theories using a ${ }^{218} \mathrm{PoO}_{x}$ cluster aerosol," Journal of Aerosol Science, vol. 21, no. 2, pp. 203-211, 1990.

[16] M. Alonso, Y. Kousaka, T. Hashimoto, and N. Hashimoto, "Penetration of nanometer-sized aerosol particles through wire screen and laminar flow tube," Aerosol Science and Technology, vol. 27, no. 4, pp. 471-480, 1997.

[17] C. A. Noble, P. A. Lawless, and C. E. Rodes, "A sampling approach for evaluating particle loss during continuous field measurement of particulate matter," Particle and Particle Systems Characterization, vol. 22, no. 2, pp. 99-106, 2005.
[18] C.-H. Jeong and G. J. Evans, "Inter-comparison of a fast mobility particle sizer and a scanning mobility particle sizer incorporating an ultrafine water-based condensation particle counter," Aerosol Science and Technology, vol. 43, no. 4, pp. 364373, 2009.

[19] Y.-H. Cheng, Z.-S. Liu, and C.-C. Chen, "On-road measurements of ultrafine particle concentration profiles and their size distributions inside the longest highway tunnel in Southeast Asia," Atmospheric Environment, vol. 44, no. 6, pp. 763-772, 2010. 


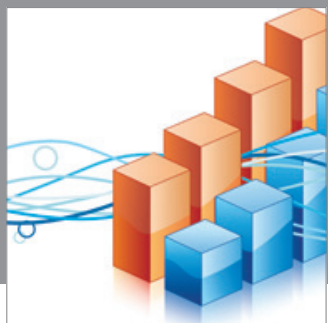

Advances in

Operations Research

mansans

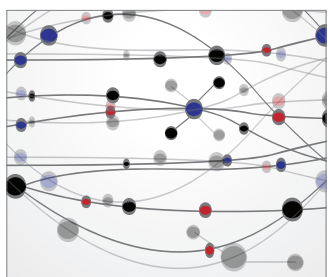

The Scientific World Journal
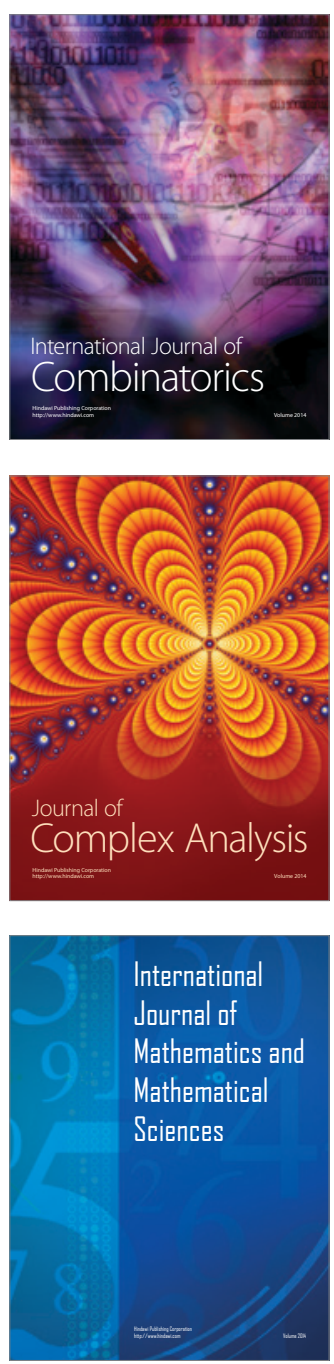
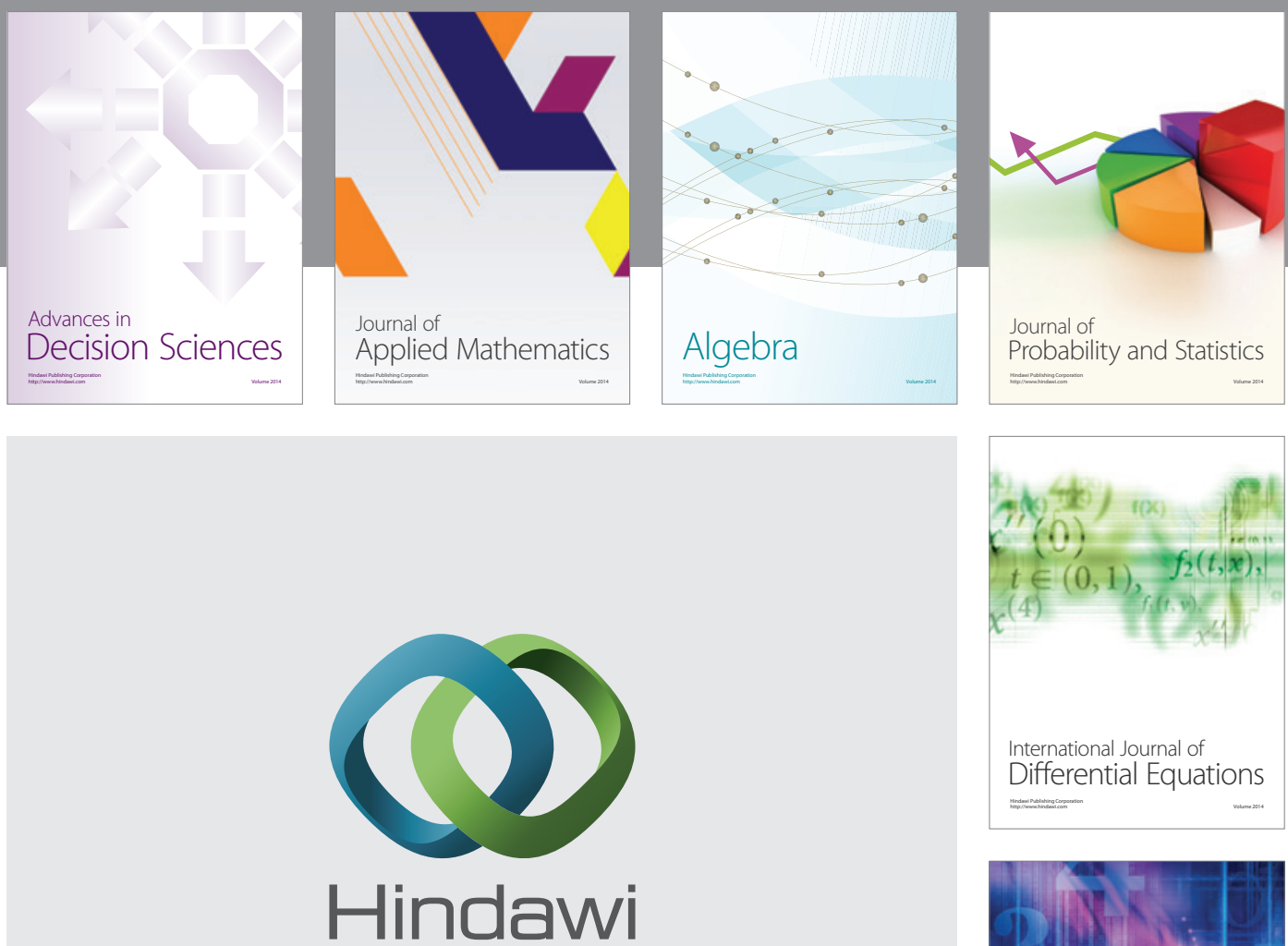

Submit your manuscripts at http://www.hindawi.com
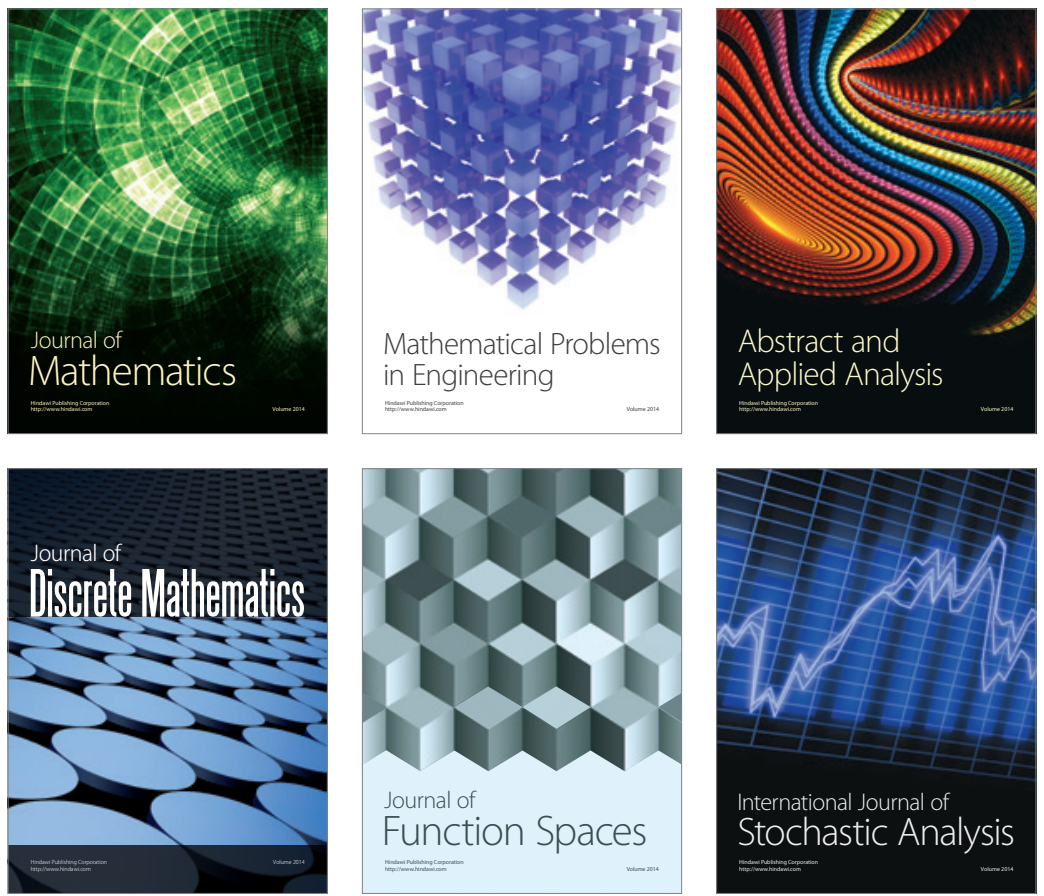

Journal of

Function Spaces

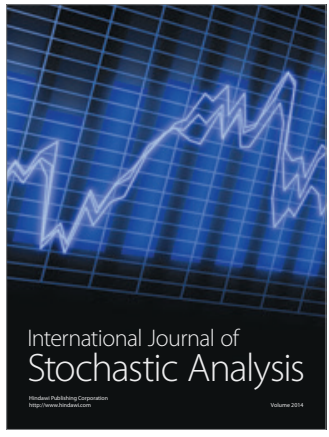

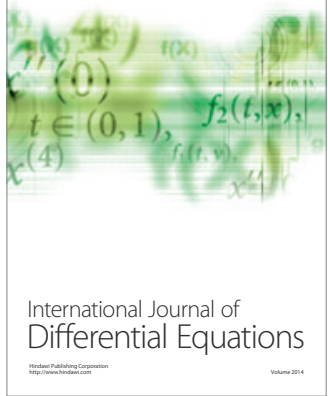
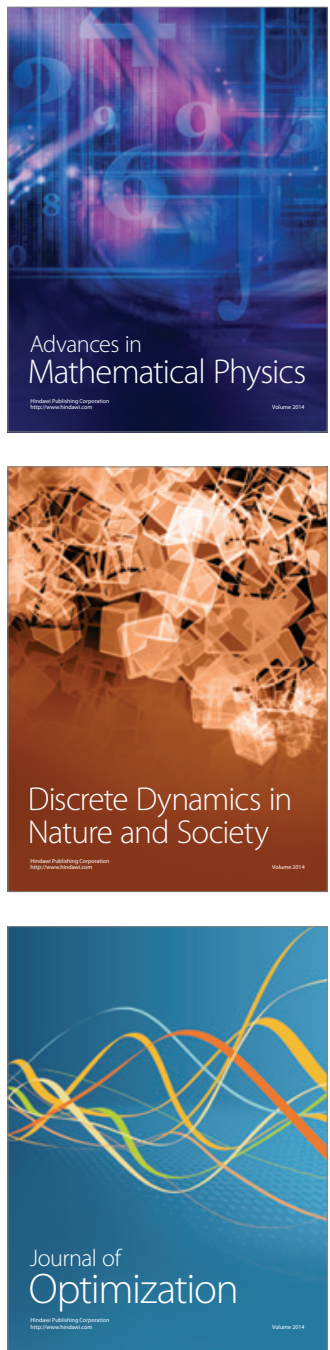